\title{
INSIDE SINGULARITY SETS OF RANDOM GIBBS MEASURES
}

\author{
JULIEN BARRAL AND STÉPHANE SEURET
}

\begin{abstract}
We evaluate the scale at which the multifractal structure of some random Gibbs measures becomes discernible. The value of this scale is obtained through what we call the growth speed in Hölder singularity sets of a Borel measure. This growth speed yields new information on the multifractal behavior of the rescaled copies involved in the structure of statistically self-similar Gibbs measures. Our results are useful to understand the multifractal nature of various heterogeneous jump processes.
\end{abstract}

\section{INTRODUCTION}

Contrary to what happens with monofractal measures (for instance uniform measures on regular Cantor sets), multifractal measures exhibit simultaneously several different behaviors at small scales. It is natural to question from which scale the multifractal structure of these measures becomes discernible and remains stable. This paper introduces a notion which provides a way to examinate the value of this critical scale. This notion, that we call growth speed in singularity sets, is naturally related with multifractal measures. In the following, we define and study the growth speed in singularity sets for a class of statistically self-similar measures which includes random Gibbs measures. This work requires refinements of the known theoretical results on the multifractal nature of these measures. Finally, we obtain rigorous estimates of the error made when approximating the asymptotic local behavior of the measure by observing it at a fine but fixed grid.

Before making precise all these notions, let us explain what one of our main motivations was. The new multifractal properties we point out in this paper are naturally involved in the small-scale structure analysis of some jump processes recently considered in [5, 8, 9]. Typical examples of such heterogeneous jump processes are Lévy processes in multifractal time. Performing a multifractal time change in irregular processes is a natural idea when trying to build multi-parameter processes [25, 27, 33].

Key words and phrases. Random Gibbs measures; Self-similarity; Large Deviations; Hausdorff dimension; Fractals. 
Indeed, such processes yield multifractal objects with an interesting structure, that may be more realistic than classical homogeneous jump processes (for instance like Lévy processes) for the purpose of modeling multifractal discontinuous phenomena (Internet traffic [23], variations of financial prices 25]). Another relevant property of these processes is that they provide new illustrations of multifractal formalisms [15, 11, 28, [5].

Our results provide tools to study these processes. Indeed, the multifractal analysis of heterogeneous jump processes in [5, 8, 9] requires to deepen our knowledge regarding statistically self-similar singular measures generated by multiplicative processes. The fact that these measures are locally equivalent to a rescaled copy of themselves is exploited in a new direction using the notion of growth speed in the Hölder singularity sets of these copies. The growth speed yields new insights on the structure of the process, which are more precise than those obtained by only considering individually these copies as the same probabilistic object. In particular, it provides a new quantitative way of distinguishing two well-known families of statistically self-similar singular measures, namely the random Gibbs measures 19 and the independent random cascades, like Mandelbrot canonical cascades 24. This paper focuses on random Gibbs measures, the case of the Mandelbrot canonical cascades is very different and treated in 7 .

The multifractal structure of random Gibbs measures has been extensively studied (15, 31, 20, 13, 29, 4, 14). This topic is concerned with the size estimation of the Hölder singularity sets of such a measure $\mu$. These sets are defined as the level sets of the pointwise Hölder exponent $\lim _{r \rightarrow 0^{+}} \frac{\log \mu(B(t, r)}{\log (r)}$. The sizes of Hölder singularity sets are measured through their Hausdorff (or packing) dimension. It can be shown that these dimensions are obtained thanks to the Legendre transform of a kind of free energy function $\tau_{\mu}$ related to $\mu$. More precisely, let $b$ be an integer $\geq 2$ and $\mathcal{A}$ the alphabet $\{0, \ldots, b-1\}$. Suppose that we are working on the symbolic space $\mathbb{A}=\mathcal{A}^{\mathbb{N}^{*}}$ endowed with the product topology and the one-sided shift transformation $\sigma$. If $w \in \mathcal{A}^{*}=\bigcup_{n \geq 1} \mathcal{A}^{n}$, the $n$ step cylinder about $w$ in $\mathbb{A}$ is denoted by $[w]$. The measures we are interested in are associated with some (random) Hölder potentiel and the dynamical system $(\mathbb{A}, \sigma)$.

The function $\tau_{\mu}$ considered in the multifractal formalism for measures in [15] 11] is obtained as follows: For every $q \in \mathbb{R}$, let

(1) $\forall j \geq 1, \tau_{\mu, j}(q)=-\frac{1}{j} \log _{b} \sum_{w \in \mathcal{A}^{j}} \mu([w])^{q}$ and $\tau_{\mu}(q)=\liminf _{j \rightarrow \infty} \tau_{\mu, j}(q)$.

The Legendre transform of $\tau_{\mu}$ at $\alpha>0$ is then $\tau_{\mu}^{*}(\alpha):=\inf _{q \in \mathbb{R}} \alpha q-\tau_{\mu}(q)$. Then the Hölder singularity set of level $\alpha>0$ is defined as

$$
E_{\alpha}^{\mu}=\left\{t \in \mathbb{A}: \lim _{n \rightarrow \infty} \frac{\log _{b} \mu([t \mid n])}{n}=\alpha\right\}
$$


$\left(t \mid n\right.$ stands for $\left.t_{1} \cdots t_{n}\right)$. The Gibbs measures we consider obey the multifractal formalism in the sense that $\operatorname{dim} E_{\alpha}^{\mu}=\tau_{\mu}^{*}(\alpha)$ when $\tau_{\mu}^{*}(\alpha)>0$.

This property is classically implied by the existence of a probability measure $\mu_{\alpha}$ of the same nature as $\mu$ and such that $\mu_{\alpha}$ is concentrated on $E_{\alpha}^{\mu} \cap E_{\tau_{\mu}^{*}(\alpha)}^{\mu_{\alpha}}$. This measure $\mu_{\alpha}$ is called an analyzing measure of $\mu$ at $\alpha$.

The existence of the measure $\mu_{\alpha}$ has another important consequence regarding the possibility of measuring how the mass of $\mu$ is distributed at a given large enough scale. Indeed, a direct consequence of the multifractal formalism (32]) and the existence of $\mu_{\alpha}$ is that for any $\varepsilon>0$ and $\alpha>0$ such that $\tau_{\mu}^{*}(\alpha)>0$, one has

(2) $\lim _{j \rightarrow \infty} \frac{\log _{b} \#\left\{w \in \mathcal{A}^{j}: b^{-j(\alpha+\varepsilon)} \leq \mu([w]) \leq b^{-j(\alpha-\varepsilon)}\right\}}{j}=\tau_{\mu}^{*}(\alpha)$.

The result we establish in this paper brings precisions on these sizes estimates. We consider a refined version of the sets $E_{\alpha}(\mu)$ by considering, for any sequence $\varepsilon_{n}$ going down to 0 , the sets

$$
\left.\widetilde{E}_{\alpha, p}^{\mu}=\left\{t \in \mathbb{A}: \forall n \geq p, b^{-n\left(\alpha+\varepsilon_{n}\right)} \leq \mu([t \mid n])\right) \leq b^{-n\left(\alpha-\varepsilon_{n}\right)}\right\},
$$

$$
\text { and } \quad \widetilde{E}_{\alpha}^{\mu}=\bigcup_{p \geq 1} E_{\alpha, p}^{\mu} .
$$

It is possible to choose $\left(\varepsilon_{n}\right)_{n>1}$ so that with probability one, for all the exponents $\alpha$ such that $\tau_{\mu}^{*}(\alpha)>0$, one has $\mu_{\alpha}\left(\widetilde{E}_{\alpha}^{\mu}\right)=\left\|\mu_{\alpha}\right\|=1$.

Since the sets sequence $\widetilde{E}_{\alpha, p}^{\mu}$ is non-decreasing and $\mu_{\alpha}\left(\widetilde{E}_{\alpha}^{\mu}\right)=1$, the growth speed $G S(\mu, \alpha)$ in $\widetilde{E}_{\alpha, p}^{\mu}$ can be defined as the smallest value of $p$ for which the $\mu_{\alpha}$-measure of $\widetilde{E}_{\alpha, p}^{\mu}$ reaches a certain positive fraction $f \in(0,1)$ of the mass of $\mu_{\alpha}$, that is the number

$$
G S(\mu, \alpha)=\inf \left\{p: \mu_{\alpha}\left(\widetilde{E}_{\alpha, p}^{\mu}\right) \geq f\left\|\mu_{\alpha}\right\|\right\} .
$$

Now for $n \geq 1$ and $\alpha>0$ let

$$
\mathcal{N}_{n}(\mu, \alpha)=\#\left\{w \in \mathcal{A}^{n}: b^{-n\left(\alpha+\varepsilon_{n}\right)} \leq \mu([w]) \leq b^{-n\left(\alpha-\varepsilon_{n}\right)}\right\} .
$$

Heuristically, one has

$$
G S(\mu, \alpha) \approx \inf \left\{p: \forall n \geq p, b^{n\left(\tau^{*}(\alpha)-\varepsilon_{n}\right)} \leq \mathcal{N}_{n}(\mu, \alpha) \leq b^{n\left(\tau^{*}(\alpha)+\varepsilon_{n}\right)}\right\},
$$

i.e. $G S(\mu, \alpha)$ controls by above the smallest rank $p$ from which considering the evaluation of $\mathcal{N}_{n}(\mu, \alpha)$ at any scale $b^{-n}$ smaller than $b^{-p}$ yields a correct representation of the asymptotic behavior of $\mathcal{N}_{n}(\mu, \alpha)$.

Our results concern estimates of the growth speed of singularities sets of copies of $\mu$ involved in the self-similarity property of $\mu$. To illustrate our purpose, let us describe the model of statistically self-similar measures 
we shall work with in the sequel. We shall consider a natural random counterpart to quasi-Bernoulli measures introduced in [26, 11] and mainly illustrated by deterministic Gibbs measures on $\mathbb{A}$. We are inspired in particular by self-similar Riesz products and their random version constructed with random phases (see 13] and examples of Section 3).

\subsection{Quasi-Bernoulli independent random measure.}

In the sequel $\equiv$ means equality in distribution.

A random probability measure $\mu=\mu(\omega)$ on $\mathbb{A}$ is said to be a quasiBernoulli independent random measure if there exists a constant $C>0$ and two sequences of random measures $\left(\mu_{j}\right)_{j \geq 1}$ and $\left(\mu^{(j)}\right)_{j \geq 1}$ such that for every $j \geq 1$,

- $(\mathbf{P} 1) \forall(v, w) \in \mathcal{A}^{j} \times \mathcal{A}^{*}, \frac{1}{C} \mu_{j}([v]) \mu^{(j)}([w]) \leq \mu([v w]) \leq C \mu_{j}([v]) \mu^{(j)}([w])$,

- (P2) for every $r \in\{0, \ldots, b-1\}, 0<\operatorname{ess} \inf \mu([r]) \leq \operatorname{ess} \sup \mu([r])<\infty$,

- $(\mathbf{P 3})\left(\mu^{(j)}([w])\right)_{w \in \mathcal{A}^{*}} \equiv(\mu([w]))_{w \in \mathcal{A}^{*}} \cdot \mu$ is also denoted $\mu^{(0)}$,

- (P4) $\sigma\left(\mu_{j}([v]): v \in \mathcal{A}^{j}\right)$ and $\sigma\left(\mu^{(j)}([w]): w \in \mathcal{A}^{*}\right)$ are independent.

The measures $\mu^{(j)}$ are the copies of $\mu$ mentioned in the paragraphs above.

1.2. Controlling the growth speed in Hölder singularity sets of the $\left(\mu^{(j)}\right)$ 's. Let $\mu$ be quasi-Bernoulli independent measure. For each copy $\mu^{(j)}$ of $\mu$, the corresponding family of analyzing measures $\mu_{\alpha}^{(j)}$ will be defined as $\mu_{\alpha}$ is defined for $\mu$. The result we focus on is the asymptotic behavior of

$$
G S\left(\mu^{(j)}, \alpha\right)=\inf \left\{N: \mu_{\alpha}^{(j)}\left(\widetilde{E}_{\alpha, N}^{\mu^{(j)}}\right) \geq f\left\|\mu_{\alpha}^{(j)}\right\|\right\} \text { as } j \rightarrow \infty .
$$

For sake of simplicity, we give in this introduction a shorter version of our main result (Theorem 2).

Theorem A. Suppose that $\tau_{\mu}$ is $C^{2}$. With probability one, for all $\alpha>0$ such that $\tau_{\mu}^{*}(\alpha)>0$ there exists $\beta>0$ such that if $j$ is large enough, $G S\left(\mu^{(j)}, \alpha\right) \leq \exp \sqrt{\beta \log j}$.

Let us introduce the quantity

$G S^{\prime}\left(\mu^{(j)}, \alpha\right)=\inf \left\{p: \forall n \geq p, b^{n\left(\tau_{\mu}^{*}(\alpha)-\varepsilon_{n}\right)} \leq \mathcal{N}_{n}\left(\mu^{(j)}, \alpha\right) \leq b^{n\left(\tau_{\mu}^{*}(\alpha)+\varepsilon_{n}\right)}\right\}$.

Theorem A also implies a control of $\mathcal{N}_{n}\left(\mu^{(j)}, \alpha\right)$ (recall (4)). A stronger version (Theorem 3) of the following result is going to be proved.

Theorem B. Suppose that $\tau_{\mu}$ is $C^{2}$. The same conclusion as in Theorem $A$ holds if $G S\left(\mu^{(j)}, \alpha\right)$ is replaced by $G S^{\prime}\left(\mu^{(j)}, \alpha\right)$.

As claimed above, Theorems A and B indeed yields new information on the multifractal structure of random Gibbs measures.

Section 2 contains new definitions and two propositions that are used in Section 3 and 5 to state and prove stronger versions of Theorems A and B. 
Section 4 contains the proof of results concerning the speed of convergence of $\tau_{\mu, j}$ to $\tau_{\mu}$.

We end this introduction by giving an application of Theorem A.

1.3. An application: The Hausdorff dimension of new limsup sets. Let $\mu$ be a quasi-Bernoulli independent random measure as defined previously and consider $\nu$, its projection on $[0,1]$. Examples of jump processes of [5, 9] are

$$
\sum_{j \geq 0} \sum_{0 \leq k \leq b^{j}-1} j^{-2} \nu\left(\left[k b^{-j},(k+1) b^{-j}\right]\right) \delta_{k b^{-j}} \text { and }(X \circ \nu([0, t]))_{0 \leq t \leq 1},
$$

where $X$ is a Lévy process. Basically, if $\left\{x_{n}\right\}$ denotes the countable set of jump points of such a process and $\left(\lambda_{n}\right)_{n \geq 1}$ is a sequence decreasing to 0 such that $\lim _{\sup _{n \rightarrow \infty}} B\left(x_{n}, \lambda_{n}\right)=[0,1]$, the multifractal nature of these processes is closely related to the computation of the Hausdorff dimension of the sets defined for every $\alpha>0, \xi>1$ by

$$
K(\alpha, \xi)=\bigcap_{N \geq 1} \bigcup_{n \geq 1: \lambda_{n}^{\alpha+\varepsilon_{n}} \leq \nu\left(\left[x_{n}-\lambda_{n}, x_{n}+\lambda_{n}\right]\right) \leq \lambda_{n}^{\alpha-\varepsilon_{n}}}\left[x_{n}-\lambda_{n}^{\xi}, x_{n}+\lambda_{n}^{\xi}\right]
$$

for some sequence $\left(\varepsilon_{n}\right)$ converging to 0 . The set $K(\alpha, \xi)$ contains the points that are infinitely often close to a jump point $x_{n}$ at rate $\xi$ relatively to $\lambda_{n}$, upon the condition that $\nu\left(\left[x_{n}-\lambda_{n}, x_{n}+\lambda_{n}\right]\right) \sim \lambda_{n}^{\alpha}$. This last condition implies that $\nu$ has roughly a Hölder exponent $\alpha$ at scale $\lambda_{n}$ around $x_{n}$. One of the main results of [5] 10] (see also 6]) is the computation of the Hausdorff dimension of $K(\alpha, \xi)$. Under a suitable assumption on $\left(\lambda_{n}\right)$, it is proved in [5] 10] that, with probability one, for all $\alpha$ such that $\tau_{\mu}^{*}(\alpha)>0$ and all $\xi \geq 1$,

$$
\operatorname{dim} K(\alpha, \xi)=\tau_{\mu}^{*}(\alpha) / \xi,
$$

where dim stands for the Hausdorff dimension. This achievement is a non-trivial generalization of what is referred to as "ubiquity" properties of the resonant system $\left\{\left(x_{n}, \lambda_{n}\right)\right\}$. Ubiquity plays a role for instance in the description of exceptional sets arising in the problem of small denominators and the physical phenomenon of resonance 1, 12. In the classical result, $\nu$ is equal to the monofractal Lebesgue measure, so $\alpha=1$, the condition $\lambda_{n}^{\alpha+\varepsilon_{n}} \leq \nu\left(\left[x_{n}-\lambda_{n}, x_{n}+\lambda_{n}\right]\right) \leq \lambda_{n}^{\alpha-\varepsilon_{n}}$ is trivial, and $\operatorname{dim} K(1, \xi)=1 / \xi$ (see 12] for instance).

The fact that, by Theorem A, the growth speed $G S\left(\mu^{(j)}, \alpha\right)$ behaves like $o(j)$ as $j \rightarrow \infty$ is a crucial issue in constructing a Cantor set of Hausdorff dimension $\tau_{\mu}^{*}(\alpha) / \xi$ in $K(\alpha, \xi)$.

\section{Definitions, Growth Speed in Singularity Sets}

In the sequel, $(\Omega, \mathcal{B}, \mathbb{P})$ denotes the probability space on which the random variables of this paper are defined. 


\subsection{Measure of singularity sets: a neighboring boxes condition.}

Let $\mu$ and $m$ be two probability measures with supports equal to $\mathbb{A}$.

With any $w \in \mathcal{A}^{n}$ can be associated the integer $i(w) \in\left\{0,1, \ldots, b^{n}-\right.$ $1\}$ such that the $b$-adic subinterval of $[0,1]$ naturally encoded by $w$ is $\left[i(w) b^{-n},(i(w)+1) b^{-n}\right]$ (alternatively $\left.i(w) b^{-n}=\sum_{k=1}^{n} w_{k} b^{-k}\right)$. Then, if $(v, w) \in \mathcal{A}^{n}, \delta(v, w)$ stands for $|i(v)-i(w)|$. This defines an integer valued distance on $\mathcal{A}^{n}$. This distance yields a notion of neighbors for cylinders of the same generation. This notion coincides with the natural one on $b$-adic subintervals of the same generation in $[0,1]$.

Let $\widetilde{\varepsilon}=\left(\varepsilon_{n}\right)_{n \geq 0}$ be a positive sequence, $N \geq 1$, and $\beta \geq 0$.

We consider a slight refinement of the sets introduced in (3): For $p \geq 1$,

$$
E_{\beta, p}^{\mu}(N, \widetilde{\varepsilon})=\left\{t \in \mathbb{A}:\left\{\begin{array}{l}
\forall n \geq p, \forall \gamma \in\{-1,1\}, \\
\forall w \in \mathcal{A}^{n}, \delta(w, t \mid n) \leq N,
\end{array} \quad b^{\gamma n\left(\beta-\gamma \varepsilon_{n}\right)} \mu([w])^{\gamma} \leq 1\right\}\right.
$$

$$
\text { and } \quad E_{\beta}^{\mu}(N, \widetilde{\varepsilon})=\bigcup_{p \geq 1} E_{\beta, p}^{\mu}(N, \widetilde{\varepsilon}) .
$$

This set contains the points $t$ for which, at each scale $n$ large enough, the $\mu$-measures of the $2 N+1$ neighbors of $[t \mid n]$ (for the distance $\delta$ ) belong to $\left[b^{-n\left(\beta+\varepsilon_{n}\right)}, b^{-n\left(\beta-\varepsilon_{n}\right)}\right]$. Controlling the mass of these neighbors is necessary in the proof of (6) when $\mu$ is a quasi-Bernoulli independent random measure.

For $n \geq 1$ and $\varepsilon, \eta>0$, let us define the quantity

$$
S_{n}^{N, \varepsilon, \eta}(m, \mu, \beta)=\sum_{\gamma \in\{-1,1\}} b^{n(\beta-\gamma \varepsilon) \gamma \eta} \sum_{v, w \in \mathcal{A}^{n}: \delta(v, w) \leq N} m([v]) \mu([w])^{\gamma \eta} .
$$

Proposition 1. Let $\left(\eta_{n}\right)_{n \geq 1}$ be a positive sequence. If $\sum_{n \geq 1} S_{n}^{N, \varepsilon_{n}, \eta_{n}}(m, \mu, \beta)<+\infty$, then $E_{\beta}^{\mu}(N, \widetilde{\varepsilon})$ is of full m-measure.

Remark 1. The same kind of conditions was used in 3 to obtain a comparison between the box [1] and centered 28] multifractal formalisms.

Proof. For $\gamma \in\{-1,1\}$ and $n \geq 1$, let us define

$$
E_{\beta}^{\mu}\left(N, \varepsilon_{n}, \gamma\right)=\left\{t \in \mathbb{A}:\left\{\begin{array}{l}
\forall w \in \mathcal{A}^{n}, \\
\delta(w, t \mid n) \leq N,
\end{array} b^{\gamma n\left(\beta-\gamma \varepsilon_{n}\right)} \mu([w])^{\gamma} \leq 1\right\} .\right.
$$

For $t \in \mathbb{A}$, if there exists (a necessarily unique) $w \in \mathcal{A}^{n}$ such that $i(w)-$ $i(t \mid n)=k$, this word $w$ is denoted $w_{k}(t)$. For $\gamma \in\{-1,1\}$, let $S_{n, \gamma}=$ $\sum_{-N \leq k \leq N} m_{k}$ with

$$
m_{k}=m\left(\left\{t \in \mathbb{A}: b^{\gamma n\left(\beta-\gamma \varepsilon_{n}\right)} \mu\left(w_{k}(t)\right)^{\gamma}>1\right\}\right) .
$$

One clearly has

$$
m\left(\left(E_{\beta}^{\mu}\left(N, \varepsilon_{n},-1\right)\right)^{c} \bigcup\left(E_{\beta}^{\mu}\left(N, \widetilde{\varepsilon}_{n}, 1\right)\right)^{c}\right) \leq S_{n,-1}+S_{n, 1},
$$


Fix $\eta_{n}>0$ and $-N \leq k \leq N$. Let $Y(t)$ be the random variable which equals $b^{\gamma n\left(\beta-\gamma \varepsilon_{n}\right) \eta_{n}} \mu\left(\left[w_{k}(t)\right]\right)^{\gamma \eta_{n}}$ if $w_{k}(t)$ exists, and 0 otherwise. The Markov inequality applied to $Y(t)$ with respect to $m$ yields $m_{k} \leq \int Y(t) d m(t)$. Since $Y$ is constant over each cylinder $[v]$ of generation $n$, we get

$$
m_{k} \leq \sum_{v, w \in \mathcal{A}^{n}: i(w)-i(v)=k} b^{n\left(\beta-\gamma \varepsilon_{n}\right) \gamma \eta_{n}} m([v]) \mu([w])^{\gamma \eta_{n}} .
$$

Summing over $|k| \leq N$ yields $S_{n,-1}+S_{n, 1} \leq S_{n}^{N, \varepsilon_{n}, \eta_{n}}(m, \mu, \beta)$. The conclusion follows from (10) and from the Borel-Cantelli Lemma.

2.2. Growth speed in families of singularity sets. Let $\Lambda$ be a set of indexes, and $\Omega^{*}$ a measurable subset of $\Omega$ of probability 1 . Some notations and technical assumptions are needed to state the result.

- For every $\omega \in \Omega^{*}$, we consider two sequences of families of measures $\left(\left\{\mu_{\lambda}^{(j)}\right\}_{\lambda \in \Lambda}\right)_{j \geq 0}$ and $\left(\left\{m_{\lambda}^{(j)}\right\}_{\lambda \in \Lambda}\right)_{j \geq 0}$ such that for every $j \geq 0$, the elements of the families $\left\{\mu_{\lambda}^{(j)}\right\}_{\lambda \in \Lambda}$ and $\left\{m_{\lambda}^{(j)}\right\}_{\lambda \in \Lambda}$ are probability measures on A. For $\nu \in\{\mu, m\},\left\{\nu_{\lambda}^{(0)}\right\}_{\lambda \in \Lambda}$ is written $\left\{\nu_{\lambda}\right\}_{\lambda \in \Lambda}$.

- We consider an integer $N \geq 1$, and a positive sequence $\widetilde{\varepsilon}=\left(\varepsilon_{n}\right)_{n \geq 1}$, as well as a family of positive numbers $\left(\beta_{\lambda}\right)_{\lambda \in \Lambda}$. Then, remembering (9) let us consider for every $j \geq 0$ and $p \geq 1$ the sets

$$
E_{\beta_{\lambda}, p}^{\mu_{\lambda}^{(j)}}(N, \widetilde{\varepsilon})=\bigcap_{n \geq p} E_{\beta_{\lambda}}^{\mu_{\lambda}^{(j)}}\left(N, \varepsilon_{n},-1\right) \cap E_{\beta_{\lambda}}^{\mu_{\lambda}^{(j)}}\left(N, \varepsilon_{n}, 1\right) .
$$

- The sets $\left\{E_{\beta_{\lambda}, p}^{\mu_{\lambda}^{(j)}}(N, \widetilde{\varepsilon})\right\}_{p}$ form a non-decreasing sequence. One then defines the growth speed of $E_{\beta_{\lambda}, p}^{\mu_{\lambda}^{(j)}}(N, \widetilde{\varepsilon})$ as the quantity

$$
G S\left(m_{\lambda}^{(j)}, \mu_{\lambda}^{(j)}, \beta_{\lambda}, N, \widetilde{\varepsilon}\right)=\inf \left\{p \geq 1: m_{\lambda}^{(j)}\left(E_{\beta_{\lambda}, p}^{\mu_{\lambda}^{(j)}}(N, \widetilde{\varepsilon})\right) \geq 1 / 2\right\}
$$

This number, maybe infinite, is a measurement of the number $p$ of generations needed for $E_{\beta_{\lambda}, p}^{\mu_{\lambda}^{(j)}}(N, \widetilde{\varepsilon})$ to recover a certain given fraction (here chosen equal to $1 / 2)$ of the probability measure $m_{\lambda}^{(j)}$. We assume that $m_{\lambda}^{(j)}$ is concentrated on $\lim _{p \rightarrow+\infty} E_{\beta_{\lambda}, p}^{\mu_{\lambda}^{(j)}}(N, \widetilde{\varepsilon})$, so that $G S\left(m_{\lambda}^{(j)}, \mu_{\lambda}^{(j)}, \beta_{\lambda}, N, \widetilde{\varepsilon}\right)<\infty$.

- We assume that for every positive sequence $\widetilde{\eta}=\left(\eta_{j}\right)_{j \geq 0}$, there exist - a random vector $V(\widetilde{\eta}) \in \mathbb{R}_{+}^{\mathbb{N}}$, a sequence $\left(V^{(j)}\right)_{j \geq 0}$ of copies of $V(\widetilde{\eta})$, - a sequence $\left(\psi_{j}(\widetilde{\eta})\right)_{j \geq 0}$ such that for $\mathbb{P}$-almost every $\omega \in \Omega^{*}$,

$$
\forall j \geq 0, \quad \forall n \geq \psi_{j}(\widetilde{\eta}), \quad V_{n}^{(j)} \geq \sup _{\lambda \in \Lambda} S_{n}^{N, \varepsilon_{n}, \eta_{n}}\left(m_{\lambda}^{(j)}, \mu_{\lambda}^{(j)}, \beta_{\lambda}\right),
$$

where $S_{n}^{N, \varepsilon_{n}, \eta_{n}}\left(m_{\lambda}^{(j)}, \mu_{\lambda}^{(j)}, \beta_{\lambda}\right)$ is defined in (8). This provides us with a uniform control over $\lambda \in \Lambda$ of the families of measures $\left(m_{\lambda}^{(j)}, \mu_{\lambda}^{(j)}\right)_{j \geq 0}$. 
Proposition 2 (Uniform growth speed in singularity sets). Let $\widetilde{\eta}=$ $\left(\eta_{j}\right)_{j \geq 0}$ be a the sequence of positive numbers.

Let $\left(\mathcal{S}_{j}\right)_{j \geq 0}$ be a sequence of integers such that $\mathcal{S}_{j} \geq \psi_{j}(\widetilde{\eta})$. Assume that

$$
\sum_{j \geq 0} \sum_{n \geq \mathcal{S}_{j}} \mathbb{E}\left(V_{n}(\widetilde{\eta})\right)<\infty
$$

With probability one, for every $j$ large enough, for every $\lambda \in \Lambda$, one has $G S\left(m_{\lambda}^{(j)}, \mu_{\lambda}^{(j)}, \beta_{\lambda}, N, \widetilde{\varepsilon}\right) \leq \mathcal{S}_{j}$.

Proof. Fix $j \geq 1$. As shown in Proposition 1 for every $n \geq 1$ and every $\lambda \in \Lambda$, one can write

$m_{\lambda}^{(j)}\left(\left(E_{\beta_{\lambda}}^{\mu_{\lambda}^{(j)}}\left(N, \varepsilon_{n},-1\right)\right)^{c} \cup\left(E_{\beta_{\lambda}}^{\mu_{\lambda}^{(j)}}\left(N, \varepsilon_{n}, 1\right)\right)^{c}\right) \leq S_{n}^{N, \varepsilon_{n}, \eta_{n}}\left(m_{\lambda}^{(j)}, \mu_{\lambda}^{(j)}, \beta_{\lambda}\right)$.

Thus, using (13), one gets

$$
m_{\lambda}^{(j)}\left(\bigcup_{n \geq \mathcal{S}_{j}}\left(E_{\beta_{\lambda}}^{\mu_{\lambda}^{(j)}}\left(N, \varepsilon_{n},-1\right)\right)^{c} \cup\left(E_{\beta_{\lambda}}^{\mu_{\lambda}^{(j)}}\left(N, \varepsilon_{n}, 1\right)\right)^{c}\right) \leq \sum_{n \geq \mathcal{S}_{j}} V_{n}^{(j)} .
$$

Now (14) yields

$$
\sum_{j \geq 1} \mathbb{P}\left(\sum_{n \geq \mathcal{S}_{j}} V_{n}^{(j)} \geq 1 / 2\right) \leq 2 \sum_{j \geq 1} \mathbb{E}\left(\sum_{n \geq \mathcal{S}_{j}} V_{n}^{(j)}\right)<\infty .
$$

Thus, with probability one, $\sum_{n}>\mathcal{S}_{j} V_{n}^{(j)}<1 / 2$ for every $j$ large enough. This, combined with (11), (15) and (12), implies that, with probability one, for all $j$ large enough, for every $\lambda \in \Lambda, G S\left(m_{\lambda}^{(j)}, \mu_{\lambda}^{(j)}, \beta_{\lambda}, N, \widetilde{\varepsilon}\right) \leq \mathcal{S}_{j}$.

\section{Main Results}

3.1. Examples of quasi-Bernoulli independent measures. It is not difficult to show that, in the setting of [19], the two following examples can be seen as random Gibbs measures associated with a random Hölder potential in the dynamical system $(\mathbb{A}, \sigma)$.

Example 1. Multinomial random measures. Let $\left(W_{0}, \ldots, W_{b-1}\right)$ be a positive random vector such that $\sum_{k=0}^{b-1} W_{j}=1$ almost surely, and let $\left(\left(W_{0}, \ldots, W_{b-1}\right)(j)\right)_{j \geq 1}$ be a sequence of independent copies of the vector $\left(W_{0}, \ldots, W_{b-1}\right)$. Let $\ell$ denote the unique measure on $\mathbb{A}$ such that $\ell([w])=b^{-n}$ for $w \in \mathcal{A}^{n}$.

With probability one, the sequence of measures $\left(\mu_{j}\right)_{j \geq 1}$ defined on $\mathbb{A}$ by

$$
\frac{d \mu_{j}}{d \ell}(t)=b^{j} \prod_{k=1}^{j} W_{w_{k}}(k) \quad\left(t \in\left[w_{1} \ldots w_{j}\right]\right)
$$


converges weakly, as $j \rightarrow \infty$, to a probability measure $\mu$ which clearly satisfies (P1) to (P4). Here $\mu^{(j)}$ is constructed like $\mu$, but with the vectors $\left(\left(W_{0}, \ldots, W_{b-1}\right)(k)\right)_{k \geq j+1}$ instead of $\left(\left(W_{0}, \ldots, W_{b-1}\right)(k)\right)_{k \geq 1}$.

Example 2. Random Riesz products. Let $\phi$ be a 1-periodic Hölder continuous function on $\mathbb{R}$ and let $\left(\theta_{k}\right)_{k \geq 0}$ be a sequence of independent random variables uniformly distributed in $[0,1]$. Let $\pi: \mathbb{A} \rightarrow[0,1]$ be the mapping $t=t_{1} \cdots t_{k} \cdots \mapsto \sum_{k \geq 1} t_{k} b^{-k}$. Then consider on $\mathbb{A}$ the sequence of measures $\left(\mu_{j}\right)_{j \geq 0}$ whose density with respect to $\ell$ is given by

$$
\frac{d \mu_{j}}{d \ell}(t)=\frac{\prod_{k=0}^{j-1} \exp \left(\phi\left(b^{k} \pi(t)+\theta_{k}\right)\right)}{\int_{0}^{1} \prod_{k=0}^{j-1} \exp \left(\phi\left(b^{k} \pi(u)+\theta_{k}\right)\right) d u} .
$$

Because of Theorems 3.1 and 3.2 in [19], with probability one, the sequence $\left\{\mu_{j}\right\}$ converges weakly to a probability measure $\mu$. Moreover, it is shown in 13, 4 that, because of the Hölder regularity and the 1-periodicity of $\phi$, properties (P1) to (P3) hold. Property (P4) follows from the fact that the $\theta_{k}$ 's are chosen independent. Here $\mu^{(j)}$ is constructed like $\mu$, but with the phases $\left(\theta_{k}\right)_{k \geq j+1}$ instead of $\left(\theta_{k}\right)_{k \geq 1}$.

\subsection{Identification of the function $\tau_{\mu}$ and auxiliary measures.}

Let $\mu$ be quasi-Bernoulli independent random measure. We specify the scaling function $\tau_{\mu}$ and the family of analysing measures discussed in the Introduction.

- The function $\tau_{\mu}$. For every $j, k \geq 1$, let us define the function

$$
\tau_{j}^{(k)}: q \in \mathbb{R} \mapsto-\frac{1}{j} \log _{b} \sum_{w \in \mathcal{A}^{j}}\left(\mu^{(k)}\right)_{j}([w])^{q},
$$

where $\left(\mu^{(k)}\right)_{j}$ denotes the measure associated with $\mu^{(k)}$ like $\mu_{j}$ is associated with $\mu$ in formulas (16) and (17). When $k=0$ we simply write $\tau_{j}(q)$.

The same arguments as those used in [13 and 4] (mainly based on Kingman's sub-multiplicative ergodic theorem) show that, with probability one, for all $q \in \mathbb{R}$ and for all $k \geq 0, \tau_{j}^{(k)}(q)$ converges, as $j \rightarrow+\infty$, to a real number $\tau_{\mu}(q)$ (thus independent of $k$ ). $\tau_{\mu}(q)$ coincides with the number defined in (1). Moreover, $\tau_{\mu}(q)$ is also the limit when $j \rightarrow+\infty$ of the sequence $\mathbb{E}\left(\tau_{j}(q)\right)$. In particular the mapping $q \mapsto \tau_{\mu}(q)$ is deterministic.

Due the concavity of $\tau_{j}$, with probability one, $\tau_{j}$ converges uniformly to $\tau_{\mu}$ on compact sets.

- Auxiliary measures. The multifractal spectrum of $\mu$ is obtained thanks to the following auxiliary measures $\mu_{q}$. Let $\Omega^{*}$ be a subset of $\Omega$ with $\mathbb{P}\left(\Omega^{*}\right)=1$ such that the conclusions of Proposition 3 hold for all $\omega \in \Omega^{*}$. For every $\omega \in \Omega^{*}$, for all $q \in \mathbb{R}$ and for all $j \geq 1$, let $\mu_{q, j}$ be the probability measure with a density with respect to the measure $\ell$ on $[v]$ (for every $\left.v \in \mathcal{A}^{j}\right)$ given by $b^{j} \mu([v])^{q} b^{j \tau_{j}(q)}$. 
If $\omega$ is still fixed, for every $q \in \mathbb{R}$ one can consider a subsequence $j_{n}(q)$ such that the sequence $\left\{\mu_{q, j_{n}(q)}\right\}_{n}$ converges weakly to a measure $\mu_{q}$ (which depends on $\omega)$. This can also be done for the measures $\mu^{(j)}$. For every fixed $\omega \in \Omega^{*}$, for all $j \geq 1$ and $q \in \mathbb{R}$, a measure $\mu_{q}^{(j)}$ is built as $\mu_{q}$.

3.3. Main results. In the sequel, $[x]$ stands for the integer part of the real number $x$. If the function $\tau_{\mu}$ is differentiable, $J$ stands for the open interval $\left\{q \in \mathbb{R}: \tau_{\mu}^{\prime}(q) q-\tau_{\mu}(q)>0\right\}$.

Theorem 1. Let $\mu$ be a quasi-Bernoulli independent random measure, and assume that $\tau_{\mu}$ is twice continuously differentiable. Let $\widetilde{\varepsilon}=\left(\varepsilon_{n}\right)_{n \geq 1}$ a sequence of positive numbers going to 0 . Assume that $\forall(M, \alpha)>0$ the series $\sum_{n>1} b^{M n^{3 / 4} \log (n)} b^{-n \alpha \varepsilon_{n}^{2}}$ converges.

With probability one, $\forall q \in J$, the singularity sets $E_{\tau_{\mu}^{\prime}(q) q-\tau_{\mu}(q)}^{\mu_{q}}(N, \widetilde{\varepsilon})$ and $E_{\tau_{\mu}^{\prime}(q)}^{\mu}(N, \widetilde{\varepsilon})$ (defined in (7)) are both of full $\mu_{q}$-measure.

Remark 2. (1) As soon as $\varepsilon_{n} \geq n^{-1 / 8} \log (n)^{1 / 2+\eta}$ for some $\eta>0$, one has $b^{M n^{3 / 4} \log (n)} b^{-n \alpha \varepsilon_{n}^{2}} \leq n^{-(1+2 \eta)}$ for all $M>0$. The conclusions of Theorem 1 thus hold in this case.

In view of the law of the iterated logarithm (see 30, 21]), one could expect $\widetilde{\varepsilon}$ to decrease faster toward 0 . This is not the case because we impose the control of neighboring cylinders (in the sense of $\delta$ ) and the uniform control over the parameter $q$.

(2) In Examples 1 and 2, $\tau_{\mu}$ is analytic (see 4] and references therein).

The next statement uses the definitions introduced in Section 2.2 The measures $\mu^{(j)}$ and $\mu_{q}^{(j)}$ play respectively the role of $\mu_{\lambda}^{(j)}$ and $m_{\lambda}^{(j)}$ for $j \geq 1$.

Theorem 2 (Growth speed in singularity sets). Under the assumptions of Theorem $\square$ let us choose $\eta>0, N \geq 1$ and a sequence $\widetilde{\varepsilon}=\left(\varepsilon_{n}\right)$ so that $\varepsilon_{n} \geq n^{-1 / 8} \log (n)^{1 / 2+\eta}$. Let us also fix $\alpha>1$.

For every compact subinterval $K$ of $J$, with probability one, for $j$ large enough and for all $q \in K$, if $\mathcal{S}_{j}=[\exp (\sqrt{\alpha \log (j)})]$, one has

$\max \left(G S\left(\mu_{q}^{(j)}, \mu^{(j)}, \tau_{\mu}^{\prime}(q), N, \widetilde{\varepsilon}\right), G S\left(\mu_{q}^{(j)}, \mu_{q}^{(j)}, \tau_{\mu}^{\prime}(q) q-\tau_{\mu}(q), N, \widetilde{\varepsilon}\right)\right) \leq \mathcal{S}_{j}$.

Remark 3. Instead of a fixed number of neighbors, it is not difficult to treat the case of an increasing sequence of neighbors $N_{n}$, simultaneously with the speed of convergence $\varepsilon_{n}$. This number $N_{n}$ can then go to $\infty$ under the condition that $\log N_{n}=o\left(n \varepsilon_{n}^{2}\right)$.

Another improvement consists in replacing the fixed fraction $f$ in (5) by a fraction $f_{j}$ going to 1 as $j$ goes to $\infty$. The choice $f_{j}=1-b^{-s_{j}}$ with $s_{j}=o([\exp (\sqrt{\alpha \log (j)})])$ is convenient, as the reader can check. 
Let us recall that for all integers $j \geq 1$ and $n \geq 1$

$$
\mathcal{N}_{n}\left(\mu^{(j)}, \alpha, \varepsilon_{n}\right)=\#\left\{w \in \mathcal{A}^{n}: b^{-n\left(\alpha+\varepsilon_{n}\right)} \leq \mu^{(j)}([w]) \leq b^{-n\left(\alpha-\varepsilon_{n}\right)}\right\} .
$$

Theorem 3 (Speed of renewal of large deviation spectrum). Under the assumptions of Theorem 1 , let us choose $\varepsilon_{n} \geq n^{-1 / 8} \log (n)^{1 / 2+\eta}$ for some $\eta>0$. Let $K$ be a compact subinterval of $J$, and let $\beta=1+$ $\max _{q \in K}|q|$.

For every $\alpha>1$, with probability one, for $j$ large enough, for all $q \in K$ and for all $n \geq[\exp (\sqrt{\alpha \log (j)})]$, one has

$$
b^{n\left(\tau_{\mu}^{\prime}(q) q-\tau_{\mu}(q)-\beta \varepsilon_{n}\right)} \leq \mathcal{N}_{n}\left(\mu^{(j)}, \tau_{\mu}^{\prime}(q), \varepsilon_{n}\right) \leq b^{n\left(\tau_{\mu}^{\prime}(q) q-\tau_{\mu}(q)+\beta \varepsilon_{n}\right)} .
$$

The following Propositions are useful in the sequel.

Proposition 3. Let $K$ be a compact subset of $\mathbb{R}$, and let us fix $\alpha>1$.

1. There exists a constant $C_{K}$ such that

$$
\text { for every } n \geq 1, \sup _{q \in K}\left|\mathbb{E}\left(\tau_{n}(q)\right)-\tau_{\mu}(q)\right| \leq C_{K} n^{-1} \text {. }
$$

2. There exists a constant $C_{K}$ such that with probability one

$$
\text { for every } n \text { large enough, } \sup _{q \in K}\left|\tau_{n}(q)-\tau_{\mu}(q)\right| \leq C_{K} \log (n) n^{-1 / 4} \text {, }
$$

and for $j$ large enough, for every $n \geq[\exp (\sqrt{\alpha \log (j)})]$,

$$
\sup _{q \in K}\left|\tau_{n}^{(j)}(q)-\tau_{\mu}(q)\right| \leq C_{K} \log (n) n^{-1 / 4} .
$$

Proposition 4. Assume that $\tau_{\mu}$ is differentiable and that $K \subset\{q \in \mathbb{R}$ : $\left.\tau_{\mu}^{\prime}(q) q-\tau_{\mu}(q)>0\right\}$. Let us denote $g_{k}$ the word consisting of $k$ consecutive zeros and $d_{k}$ the word consisting of $k$ consecutive $b-1$.

There are three constants $\left(C, \eta_{0}, \Lambda\right) \in \mathbb{R}_{+}^{* 3}$ such that with probability one,

$$
\sup _{\substack{q \in K, n \geq 0 \\ \gamma \in\{-1,1\}, \eta \in\left(0, \eta_{0}\right]}}\left(\mu_{q}\left(\left[d_{n}\right]\right) \frac{\mu\left(\left[g_{n}\right]\right)^{\gamma \eta}}{\mu\left(\left[d_{n}\right]\right)^{\gamma \eta}}+\mu_{q}\left(\left[g_{n}\right]\right) \frac{\mu\left(\left[d_{n}\right]\right)^{\gamma \eta}}{\mu\left(\left[g_{n}\right]\right)^{\gamma \eta}}\right) b^{n \Lambda} \leq C,
$$

and for $j$ large enough, for every $n \geq[\exp (\sqrt{\alpha \log (j)})]$,

$$
\sup _{\substack{q \in K, \gamma \in\{-1,1\}, \eta \in\left(0, \eta_{0}\right]}}\left(\mu_{q}^{(j)}\left(\left[d_{n}\right]\right) \frac{\mu^{(j)}\left(\left[g_{n}\right]\right)^{\gamma \eta}}{\mu^{(j)}\left(\left[d_{n}\right]\right)^{\gamma \eta}}+\mu_{q}^{(j)}\left(\left[g_{n}\right]\right) \frac{\mu^{(j)}\left(\left[d_{n}\right]\right)^{\gamma \eta}}{\mu^{(j)}\left(\left[g_{n}\right]\right)^{\gamma \eta}}\right) b^{n \Lambda} \leq C .
$$

Propositions 3 and 4 are proved in Section 4 and the theorems in Section 5 


\section{Proofs of Proposition 3 and 4}

4.1. Proof of Proposition 3. 1. The arguments are standard. For $q \in \mathbb{R}$ and $j \geq 1$, let us define $L_{j}(q)=j \mathbb{E}\left(\tau_{j}(q)\right)$. As a consequence of (P1),

$$
\begin{aligned}
C^{-|q|} \sum_{v \in \mathcal{A}^{j}, w \in \mathcal{A}^{n}}\left(\mu_{j}([v]) \mu^{(j)}([w])\right)^{q} & \leq \sum_{v \in \mathcal{A}^{j+n}} \mu([v])^{q} \\
\text { and } \sum_{v \in \mathcal{A}^{j+n}} \mu([v])^{q} & \leq C^{|q|} \sum_{v \in \mathcal{A}^{j}, w \in \mathcal{A}^{n}}\left(\mu_{j}([v]) \mu^{(j)}([w])\right)^{q} .
\end{aligned}
$$

Using then (P3) and (P4), and the definition of $\tau_{j}(q)$, one gets

$$
\forall j, n \geq 1, \forall q \in \mathbb{R},\left|L_{j+n}(q)-L_{j}(q)-L_{n}(q)\right| \leq C_{q}:=|q| \log _{b}(C) .
$$

It follows that the two sequences $L_{j}(q)+C_{q}$ and $-L_{j}(q)+C_{q}$ are subadditive. Consequently, the sequence $\left(L_{j}(q)+C_{q}\right) / j$ converges, as $j \rightarrow+\infty$, to its infimum denoted by $L(q)$. Similarly, the sequence $\left(-L_{j}(q)+C_{q}\right) / j$ converges to $-L(q)$. This yields that

$$
\forall j \geq 1, \forall q \in \mathbb{R},\left|L_{j}(q) / j-L(q)\right| \leq C_{q} / j,
$$

which gives the desired conclusion since we have seen that $L(q)=\tau_{\mu}(q)$.

2. We invoke a property which does hold because of (P2): there exists $M>0$ such that with probability one,

$$
\forall q, q^{\prime} \in \mathbb{R}^{2}, \forall j \geq 1,\left|\tau_{j}(q)-\tau_{j}\left(q^{\prime}\right)\right| \leq M\left|q-q^{\prime}\right| .
$$

Fix $K$, a non-trivial compact subinterval of $\mathbb{R}$.

For $q \in K, j \geq 0$ and $n \geq 1$, let us define the random variables

$$
L_{n}^{(j)}(q)=-\log _{b} \sum_{w \in \mathcal{A}^{n}}\left(\mu^{(j)}\right)_{n}([w])^{q},
$$

and $L_{n}(q)=L_{n}^{(0)}(q)$. It follows from (P1) and (P4) that

(21) $\forall q \in K, \forall j, n \geq 1, \forall q \in K,\left|L_{j+n}(q)-L_{j}(q)-L_{n}^{(j)}(q)\right| \leq|q| \log _{b}(C)$.

Let $C_{K}=\sup _{q \in K}|q| \log _{b}(C)$, and fix $q \in K$. For every integer $m \geq 1$, we write $m=[\sqrt{m}]^{2}+i_{m}$ where $i_{m} \in[0,3 \sqrt{m}]$. Using again $(\mathbf{P 1})$ to $(\mathbf{P} 4)$, one deduces from (21) that for every $m \geq 1$, there exist $[\sqrt{m}]$ independent copies $X_{1}^{(m)}, \ldots, X_{[\sqrt{m}]}^{(m)}$ of $L_{[\sqrt{m}]}(q)$ such that

$$
\left|L_{m}(q)-\sum_{i=1}^{[\sqrt{m}]} X_{i}^{(m)}(q)\right| \leq C_{K}[\sqrt{m}]+\left|L_{i_{m}}(q)\right| \leq 4 C_{K} \sqrt{m} .
$$

We invoke the following concentration inequality (see Lemma 1.5 of [22]) 
Lemma 1. Let $n \geq 1$ and let $\left(Y_{i}\right)_{1 \leq i \leq n}$ be a sequence of random variable i.i.d. with a centered and bounded random variable $Y$. For all $s>0$,

$$
\mathbb{P}\left(\left|\sum_{i=1}^{n} Y_{i}\right|>\|Y\|_{\infty} s \sqrt{n}\right) \leq 2 \exp \left(-s^{2} / 2\right)
$$

Let us define the random variables $Y_{i}^{(m)}(q)=X_{i}^{(m)}(q)-\mathbb{E}\left(X_{i}^{(m)}(q)\right)$. By (P2), one can find a constant $M_{K}>0$ such that $\sup _{q \in K}\left|Y_{i}^{m}(q)\right| \leq$ $M_{K}[\sqrt{m}]$. As a consequence, Lemma 1 can be applied to the bounded family $Y_{1}^{(m)}(q), \ldots, Y_{[\sqrt{m}]}^{(m)}(q)$. Then choosing $s=\sqrt{2} \log (m)$ yields (remember that $\left.\|Y\|_{\infty} \leq M_{K}[\sqrt{m}]\right)$

$$
\mathbb{P}\left(\left|\sum_{i=1}^{[\sqrt{m}]} Y_{i}^{(m)}(q)\right|>\sqrt{2} M_{K} \log (m)[\sqrt{m}]^{3 / 2}\right) \leq \exp \left(-(\log m)^{2}\right) .
$$

For every $m \geq 1$, let $q_{1}^{(m)}<q_{2}^{(m)}<\cdots<q_{k}^{(m)}<\ldots$ be a finite sequence of points of $K$ such that $q_{k+1}^{(m)}-q_{k}^{(m)} \leq m^{-1 / 4}$, and denote by $\mathcal{R}_{m}$ the set of these points. We can assume that the cardinality of $\mathcal{R}_{m}$ is less than or equal to $|K| \sqrt{m+1}$. Then

$$
\begin{aligned}
& \sum_{m \geq 1} \mathbb{P}\left(\exists q \in \mathcal{R}_{m},\left|\sum_{i=1}^{[\sqrt{m}]} Y_{i}^{(m)}(q)\right|>\sqrt{2} M_{K} \log (m)[\sqrt{m}]^{3 / 2}\right) \\
\leq & \sum_{m \geq 1}|K| \sqrt{m+1} \exp \left(-(\log m)^{2}\right)<\infty .
\end{aligned}
$$

This implies that for every $q \in \mathcal{R}_{m}$ and for $m$ large enough,

$$
\left|\sum_{i=1}^{[\sqrt{m}]} Y_{i}^{(m)}(q)\right| \leq \sqrt{2} \log m M_{K}[\sqrt{m}]^{3 / 2} .
$$

On the other hand, remembering the proof of item 1. and (19), one has

$$
\forall q \in \mathcal{R}_{m},\left|\mathbb{E}\left(X_{1}^{m}(q)\right)-[\sqrt{m}] \tau_{\mu}(q)\right| \leq C_{K} .
$$

For every $q \in \mathcal{R}_{m},\left|L_{m}(q)-m \tau_{\mu}(q)\right|$ can be upper bounded by

$$
\begin{array}{r}
\left|L_{m}(q)-\sum_{i=1}^{[\sqrt{m}]} X_{i}^{(m)}(q)\right|+\left|\sum_{i=1}^{[\sqrt{m}]} Y_{i}^{(m)}(q)\right| \\
+\left|\sum_{i=1}^{[\sqrt{m}]} \mathbb{E}\left(X_{i}^{(m)}(q)\right)-[\sqrt{m}]^{2} \tau_{\mu}(q)\right|+i_{m}\left|\tau_{\mu}(q)\right| .
\end{array}
$$

With probability one, for $m$ large enough, using respectively (22) and (24), this first and the third term are both bounded by a $O([\sqrt{m}])$ (which does 
not depend on $q)$. Using (23) and remarking that $i_{m}=O([\sqrt{m}])$, one gets

$$
\left|L_{m}(q)-m \tau_{\mu}(q)\right| \leq \sqrt{2} M_{K} \log (m)[\sqrt{m}]^{3 / 2}+O([\sqrt{m}]),
$$

where $O([\sqrt{m}])$ is uniform over $q \in \mathcal{R}_{m}$. This yields $\left|\tau_{m}(q)-\tau_{\mu}(q)\right|=$ $O\left(\log (m) m^{-1 / 4}\right)$ uniformly for $q \in \mathcal{R}_{m}$ when $m$ is large enough. The conclusion follows from (20) and from the construction of the sets $\mathcal{R}_{m}$.

Let us show the second inequality of item 2. For every $j \geq 0, m \geq 1$ and $q \in K$, let us consider a sequence $Y_{i}^{(m), j}(q), 1 \leq i \leq[\sqrt{m}]$, associated with $\mu=\mu^{(j)}$ like $Y_{i}^{(m)}(q), 1 \leq i \leq[\sqrt{m}]$, is associated with $\mu=\mu^{(0)}$. Let $\mathcal{R}_{m}$ be defined as above, and let us consider the events

$$
A(j, m)=\left\{\exists q \in \mathcal{R}_{m},\left|\sum_{i=1}^{[\sqrt{m}]} Y_{i}^{(m), j}(q)\right|>\sqrt{2} M_{K} \log (m)[\sqrt{m}]^{3 / 2}\right\} .
$$

One verifies that $\sum_{j \geq 0} \sum_{m \geq[\exp \sqrt{\alpha \log (j)}]} \mathbb{P}(A(j, m))<\infty$. We then deduce from the Borel-Cantelli Lemma that with probability one, for $j$ large enough, if $m \geq[\exp (\sqrt{\alpha \log (j)})]$ then $A(j, m)^{c}$ holds. One concludes by using the same estimates as above.

4.2. Proof of Proposition 4. If $t_{j} \in\left\{g_{j}, d_{j}\right\}$, the same kind of arguments as in the proof of Proposition 3 show that, with probability one, $\Lambda_{t}(1)=\lim _{j \rightarrow \infty} \frac{1}{j} \log _{b}\left(\mu\left(\left[t_{j}\right]\right)\right)$ exists, and this number is deterministic. Hence, using (25), with probability one, for every $q \in \mathbb{R}$, the limit $\Lambda_{t}(q)=\lim _{j \rightarrow \infty} \frac{1}{j} \log _{b}\left(\mu_{q}\left(\left[t_{j}\right]\right)\right)$ exists and is equal to $q \Lambda_{t}(1)+\tau_{\mu}(q)$. Since $\mu_{q}$ is a finite measure, $\Lambda_{t}(q) \leq 0$.

Moreover, there exists $C_{K}>0$ such that for $\mathbb{P}$-almost every $\omega \in \Omega^{*}$, for $j$ large enough, for all $q \in K \cup\{1\}$,

$$
\begin{aligned}
& \left|\frac{1}{j} \log _{b}\left(\mu_{q}\left(\left[t_{j}\right]\right)\right)-\Lambda_{t}(q)\right| \leq C_{K} \log (j) j^{-1 / 4} \\
\forall k \geq[\exp (\sqrt{\alpha \log (j)})], \quad & \left|\frac{1}{k} \log _{b}\left(\mu_{q}^{j}\left(\left[t_{k}\right]\right)\right)-\Lambda_{t}(q)\right| \leq C_{K} \log (k) k^{-1 / 4} .
\end{aligned}
$$

So, for $j$ large enough, $\gamma \in\{-1,1\}$ and $\eta>0$, one has

$$
\mu_{q}\left(\left[d_{j}\right]\right) \frac{\mu\left(\left[g_{j}\right]\right)^{\gamma \eta}}{\mu\left(\left[d_{j}\right]\right)^{\gamma \eta}}+\mu_{q}\left(\left[g_{j}\right]\right) \frac{\mu\left(\left[d_{j}\right]\right)^{\gamma \eta}}{\mu\left(\left[g_{j}\right]\right)^{\gamma \eta}} \leq f(j)
$$

and $\forall k \geq[\exp (\sqrt{\alpha \log (j)})]$

$$
\mu_{q}^{(j)}\left(\left[d_{k}\right]\right) \frac{\mu^{(j)}\left(\left[g_{k}\right]\right)^{\gamma \eta}}{\mu^{(j)}\left(\left[d_{k}\right]\right)^{\gamma \eta}}+\mu_{q}^{(j)}\left(\left[g_{k}\right]\right) \frac{\mu^{(j)}\left(\left[d_{k}\right]\right)^{\gamma \eta}}{\mu^{(j)}\left(\left[g_{k}\right]\right)^{\gamma \eta}} \leq f(k),
$$

where

$$
f(j)=b^{(2 \eta+1) C_{K} j^{3 / 4} \log (j)} b^{j \eta\left(\left|\Lambda_{d}(1)\right|+\left|\Lambda_{g}(1)\right|\right)}\left(b^{j \Lambda_{d}(q)}+b^{j \Lambda_{g}(q)}\right) .
$$


Let us show that $\Lambda_{t}(q)<0$ for $t \in\{g, d\}$ and $q \in K$. Suppose $\Lambda_{t}\left(q_{0}\right)=0$ for some $q_{0} \in K$. Remember that $q \Lambda_{t}(1)+\tau_{\mu}(q)=\Lambda_{t}(q) \leq 0$ for all $q \in \mathbb{R}$. Using the concavity of $\tau_{\mu}$, the equality $\Lambda_{t}\left(q_{0}\right)=0$ implies that $\tau_{\mu}^{\prime}\left(q_{0}\right)=-\Lambda_{t}(1)$ and then that $\tau_{\mu}^{\prime}\left(q_{0}\right) q_{0}-\tau_{\mu}\left(q_{0}\right)=0$, in contradiction with our assumption $K \subset J$.

Finally, since $\Lambda_{t}(q)=q \Lambda_{t}(1)+\tau_{\mu}(q)$, the mapping $q \mapsto \Lambda_{t}(q)$ is continuous, and the conclusion follows from properties (P1) and (P2), the compactness of $K$, and the form of $f(j)$.

\section{Proofs of Theorems 1, 2] and 3}

Mimicking the approach in 4 and using Proposition 3 and 4 shows that if $K$ is a compact subset of $\mathbb{R}$, there exists a constant $M_{K}$ such that, with probability one, for $n$ large enough, $\forall v \in \mathcal{A}^{n}, \forall q \in K$,

$$
M_{K}^{-1} b^{-\left(M_{K}\right) n^{3 / 4} \log (n)} \leq \frac{\mu_{q}([v])}{\mu([v])^{q} b^{n \tau_{\mu}(q)}} \leq M_{K} b^{\left(M_{K}\right) n^{3 / 4} \log (n)} .
$$

and if $\alpha>1$ is fixed, for the same constant $M_{K}$, with probability one, for $j$ large enough, for $n \geq[\exp (\sqrt{\alpha \log (j)})], \forall v \in \mathcal{A}^{n}, \forall q \in K$,

$$
M_{K}^{-1} b^{-\left(M_{K}\right) n^{3 / 4} \log (n)} \leq \frac{\mu_{q}^{(j)}([v])}{\mu^{(j)}([v])^{q} b^{n \tau_{\mu}(q)}} \leq M_{K} b^{\left(M_{K}\right) n^{3 / 4} \log (n)} .
$$

Before starting the proofs, let us make a last useful remark.

Remark 4. If $v$ and $w$ are words of length $n$, and if $\bar{v}$ and $\bar{w}$ stand for their prefixes of length $n-1$, then $\delta(\bar{v}, \bar{w})>k$ implies $\delta(v, w)>b k$. It implies that, given two integers $n \geq m>0$ and two words $v$ and $w$ in $\mathcal{A}^{n}$ such that $b^{m-1}<\delta(v, w) \leq b^{m}$, there are two prefixes $\bar{v}$ and $\bar{w}$ of respectively $v$ and $w$ of common length $n-m$ such that $\delta(\bar{v}, \bar{w}) \leq 1$; moreover, for these words $\bar{v}$ and $\bar{w}$, there are at most $b^{2 m}$ pairs $(v, w)$ of words in $\mathcal{A}^{n}$ such that $\bar{v}$ and $\bar{w}$ are respectively the prefixes of $v$ and $w$.

5.1. Proof of Theorem 1, Fix $K$ a compact subinterval of $J$ and $\left(\eta_{n}\right)_{n \geq 1}$ a bounded positive sequence to be precised later. For $\omega \in \Omega^{*}$ and $q \in K$, let us introduce the two quantities (recall (8) )

$F_{n}(q)=S_{n}^{N, \varepsilon_{n}, \eta_{n}}\left(\mu_{q}, \mu, \tau_{\mu}^{\prime}(q)\right)$ and $G_{n}(q)=S_{n}^{N, \varepsilon_{n}, \eta_{n}}\left(\mu_{q}, \mu_{q}, \tau_{\mu}^{\prime}(q) q-\tau_{\mu}(q)\right)$.

Due to Proposition 1] we seek for a uniform control of $F_{n}$ and $G_{n}$ on $K$. We only consider $F_{n}$, since the study of $G_{n}$ is similar.

- An upper bound for $F_{n}(q)$ : Consider $v, w \in \mathcal{A}^{n}$ such that $\delta(v, w)=$ $k \leq N$, as well as two prefixes $\bar{v}$ and $\bar{w}$ of respectively $v$ and $w$ of common length $n-\left[\log _{b}(k)\right]$ such that $\delta(\bar{v}, \bar{w}) \leq 1$. Let $q \in K$. If $n$ is large enough, (25) holds for both $v$ and $\bar{v}$. Then, using the construction of $\mu_{q}$, item $\mathbf{2}$. of 
Proposition 3] (P1), (P2) and (25), one gets for $n$ large enough

$$
\mu_{q}([v]) \leq \widetilde{C} b^{\widetilde{C} n^{3 / 4} \log (n)} \mu_{q}([\bar{v}]) \quad \text { and } \quad \mu([w])^{\gamma \eta_{n}} \leq \widetilde{C} \mu([\bar{w}])^{\gamma \eta_{n}},
$$

where $\widetilde{C}$ depends on $C, K,\|\widetilde{\eta}\|_{\infty}$ and $\|\widetilde{\varepsilon}\|_{\infty}$. Thus, by Remark 4 for $n$ large enough, $0 \leq k \leq N$ and $\gamma \in\{-1,1\}$,

$$
\begin{aligned}
& b^{n\left(\tau_{\mu}^{\prime}(q)-\gamma \varepsilon_{n}\right) \gamma \eta_{n}} \sum_{v, w \in \mathcal{A}^{n}, \delta(v, w)=k} \mu_{q}([v]) \mu([w])^{\gamma \eta_{n}} \\
\leq & \widetilde{C} b^{\widetilde{C} n^{3 / 4} \log (n)} b^{\left(n-\left[\log _{b} k\right]\right)\left(\tau_{\mu}^{\prime}(q)-\gamma \varepsilon_{n}\right) \gamma \eta_{n}} \sum_{v, w \in \mathcal{A}^{n-\left[\log _{b} k\right], \delta(v, w) \leq 1}} \mu_{q}([v]) \mu([w])^{\gamma \eta_{n}},
\end{aligned}
$$

for some other constant $\widetilde{C}$ depending on $C, K, N,\|\widetilde{\varepsilon}\|_{\infty}$ and $\|\widetilde{\eta}\|_{\infty}$.

Let us remark that for every integer $l \in\left\{0, . ., \log _{b}(N)\right\}$, there are less than $b^{l+1}$ integers $k \in[0, N]$ such that $\left[\log _{b} k\right]=l$. One thus deduces from the definition (27) (and (8)) of $F_{n}(q)$ and from the above estimate that

$$
F_{n}(q) \leq \widetilde{C} b^{\widetilde{C} n^{3 / 4} \log (n)} \sum_{l=0}^{\left[\log _{b}(N)\right]+1} b^{l+1}\left(T_{1}(q, n, l)+T_{2}(q, n, l)\right),
$$

where

$$
\begin{aligned}
& \text { (30) } T_{1}(q, n, l)=\sum_{\gamma \in\{-1,1\}} b^{(n-l)\left(\tau_{\mu}^{\prime}(q)-\gamma \varepsilon_{n}\right) \gamma \eta_{n}} \sum_{w \in \mathcal{A}^{n-l}} \mu_{q}([w]) \mu([w])^{\gamma \eta_{n}} \\
& \text { (31) } T_{2}(q, n, l)=\sum_{\gamma \in\{-1,1\}} b^{(n-l)\left(\tau_{\mu}^{\prime}(q)-\gamma \varepsilon_{n}\right) \gamma \eta_{n}} \sum_{v, w \in \mathcal{A}^{n-l}, \delta(v, w)=1} \mu_{q}([v]) \mu([w])^{\gamma \eta_{n}} .
\end{aligned}
$$

Let us first upper bound $T_{1}(q, n, l)$. (25) yields for some constant $M_{K}^{\prime}$ that

$$
\sum_{w \in \mathcal{A}^{m}} \mu_{q}([w]) \mu([w])^{\gamma \eta_{n}} \leq b^{m \tau_{\mu}(q)} M_{K}^{\prime} b^{M_{K}^{\prime} m^{3 / 4} \log m} \sum_{w \in \mathcal{A}^{m}} \mu([w])^{q+\gamma \eta_{n}},
$$

where $m=n-l$. Using item 2. of Proposition 3 for some constant $C_{K}$

$$
\sum_{w \in \mathcal{A}^{n-l}} \mu([w])^{q+\gamma \eta_{n}} \leq b^{-(n-l) \tau_{\mu}\left(q+\gamma \eta_{n}\right)+(n-l) C_{K} \log (n-l)(n-l)^{-1 / 4}} .
$$

Since $\tau_{\mu}$ is twice continuously differentiable, one has $\tau_{\mu}\left(q+\gamma \eta_{n}\right)-\tau_{\mu}(q)-$ $\gamma \eta_{n} \tau_{\mu}^{\prime}(q)=\eta_{n} O\left(\eta_{n}\right)$ independently of $q \in K$ (if $\|\widetilde{\eta}\|$ is small enough), and

$$
T_{1}(q, n, l) \leq 2 M_{K}^{\prime} b^{\left(M_{K}^{\prime}+C_{K}\right)(n-l)^{3 / 4} \log (n-l)} b^{-(n-l) \eta_{n}\left(\varepsilon_{n}+O\left(\eta_{n}\right)\right)} .
$$

In order to estimate $T_{2}(q, n, l)$, we use the words $g_{k}$ and $d_{k}$ defined in Proposition 4 For every $m \geq 1$, a representation of the set of pairs $(v, w)$ in $\mathcal{A}^{m}$ such that $\imath(w)=\imath(v)+1$ is the following:

$$
\bigcup_{k=0}^{m-1} \bigcup_{u \in \mathcal{A}^{m-1-k}} \bigcup_{r \in\{0, \ldots, b-2\}}\left\{\left(u \cdot r \cdot d_{k}, u \cdot(r+1) \cdot g_{k}\right)\right\} \text {. }
$$


Let $m=n-l$. The $\operatorname{sum} \mathcal{T}_{n, \gamma}(q)=\sum_{v, w \in \mathcal{A}^{m}, \delta(v, w)=1} \mu_{q}([v]) \mu([w])^{\gamma \eta_{n}}$ equals

$$
\sum_{k=0}^{m-1} \sum_{u \in \mathcal{A}^{m-1-k}} \sum_{r=0}^{b-2} \sum_{(e, f) \in\{(d, g),(g, d)\}} \mu_{q}\left(\left[u . r . e_{k}\right]\right) \mu\left(\left[u .(r+1) . f_{k}\right]\right)^{\gamma \eta_{n}} .
$$

Let us introduce $\Theta(q, k, n, \gamma)=\mu\left(\left[d_{k}\right]\right)^{q} \mu\left(\left[g_{k}\right]\right)^{\gamma \eta_{n}}+\mu\left(\left[g_{k}\right]\right)^{q} \mu\left(\left[d_{k}\right]\right)^{\gamma \eta_{n}}$. Using (25) and property (P1) of $\mu$, one obtains another constant $\widetilde{C}$ such that

$$
\mathcal{T}_{n, \gamma}(q) \leq \widetilde{C}(b-2) b^{C_{K} m^{3 / 4} \log (m)} b^{m \tau_{\mu}(q)} \sum_{k=0}^{m-1} \Theta(q, k, n, \gamma) \sum_{u \in \mathcal{A}^{m-1-k}} \mu([u])^{q+\gamma \eta_{n}} .
$$

Then, item 2. of Proposition 3 yields (with another $\widetilde{C}$ )

$$
\begin{aligned}
& \mathcal{T}_{n, \gamma}(q) \\
\leq & \widetilde{C} b^{C_{K} m^{3 / 4} \log (m)} b^{m \tau_{\mu}(q)} \\
& \quad \times \sum_{k=0}^{m-1} \Theta(q, k, n, \gamma) b^{2 C_{K}(m-1-k)^{3 / 4} \log (m-k-1)-(m-k-1) \tau_{\mu}\left(q+\gamma \eta_{n}\right)} \\
\leq & \widetilde{C} b^{2 C_{K} m^{3 / 4} \log (m)} b^{m\left(\tau_{\mu}(q)-\tau_{\mu}\left(q+\gamma \eta_{n}\right)\right)} \sum_{k=0}^{m-1} \Theta(q, k, n, \gamma) b^{(k+1) \tau_{\mu}\left(q+\gamma \eta_{n}\right)} \\
\leq & \widetilde{C} b^{m\left(-\tau_{\mu}^{\prime}(q) \gamma \eta_{n}+O\left(\eta_{n}^{2}\right)\right)} b^{2 C_{K} m^{3 / 4} \log (m)} \sum_{k=0}^{m-1} \Theta(q, k, n, \gamma) b^{k \tau_{\mu}\left(q+O\left(\eta_{n}\right)\right)} .
\end{aligned}
$$

By Proposition 4 and (25), the sum $\sum_{k=0}^{m-1} \Theta(q, k, n, \gamma) b^{k \tau_{\mu}\left(q+O\left(\eta_{n}\right)\right)}$ is uniformly bounded over $q \in K$ and $m \geq 0$ when $\|\widetilde{\eta}\|_{\infty}$ is small enough. Hence, replacing $m$ by $n-l$,

$$
T_{2}(q, n, l) \leq \widetilde{C} b^{2 C_{K}(n-l)^{3 / 4} \log (n-l)} b^{-(n-l) \eta_{n}\left(\varepsilon_{n}+O\left(\eta_{n}\right)\right)} .
$$

Finally, combining (29), (32) and (34) yields

$$
\begin{aligned}
F_{n}(q) & \leq \widetilde{C} b^{\widetilde{C} n^{3 / 4} \log (n)} \sum_{l=0}^{\left[\log _{b}(N)\right]+1} b^{l+1} b^{O\left((n-l)^{3 / 4} \log (n-l)\right)} b^{-(n-l) \eta_{n}\left(\varepsilon_{n}+O\left(\eta_{n}\right)\right)} \\
& =O\left(b^{M n^{3 / 4} \log (n)} b^{-n \eta_{n}\left(\varepsilon_{n}+O\left(\eta_{n}\right)\right)}\right)
\end{aligned}
$$

for some $M>0$ independently of $q \in K$. By our assumption on $\varepsilon_{n}$, the choice $\eta_{n}=\alpha \varepsilon_{n}$ with $\alpha$ small enough so that $\eta_{n}\left(\varepsilon_{n}+O\left(\eta_{n}\right)\right) \geq \alpha \varepsilon_{n}^{2} / 2$ makes the series $\sum_{n>1} F_{n}(q)$ converge for every $q \in K$. The conclusion concerning the sets $E_{\tau_{\mu}^{\prime}(q)}^{\mu}(N, \widetilde{\varepsilon})$ then follows from Proposition 1 
5.2. Proof of Theorem 2, Fix $\bar{\alpha} \in(1, \alpha)$. We use twice (26), with $\bar{\alpha}$ and $\alpha$, in order to get a control like (28). For $j$ large enough, if $n \geq$ $[\exp (\sqrt{\alpha \log (j)})]$ and if $v \in \mathcal{A}^{n}$ and $\bar{v}$ is a prefix of $v$ such that $|\bar{v}| \geq$ $n-\log _{b}(n)$, then $|\bar{v}| \geq[\exp (\sqrt{\bar{\alpha} \log (j)})]$ and (28) holds for $v$ and $\bar{v}$. Then, from the computations performed in the proof of Theorem 1 and from Proposition 4 one deduces that for every compact subinterval $K$ of $J$, there exist $C, M, \beta>0$ and $\widetilde{\eta}=\left(\eta_{n}\right)_{n \geq 1} \in \mathbb{R}_{+}^{\mathbb{N}^{*}}$ such that with probability one, for $j$ large enough, if $n \geq[\exp (\sqrt{\alpha \log (j)})]$, for all $q \in K$

$$
\begin{aligned}
& \max \left(S^{N, \varepsilon_{n}, \eta_{n}}\left(\mu_{q}^{(j)}, \mu^{(j)}, \tau_{\mu}^{\prime}(q)\right), S^{N, \varepsilon_{n}, \eta_{n}}\left(\mu_{q}^{(j)}, \mu_{q}^{(j)}, q \tau_{\mu}^{\prime}(q)-\tau_{\mu}(q)\right)\right) \\
& \leq C b^{M n^{3 / 4} \log (n)-\beta n \varepsilon_{n}^{2}} .
\end{aligned}
$$

In order to apply Proposition 2 let us define

- $\Lambda=K, \lambda=q$ and $\left\{\left(m_{\lambda}^{(j)}, \mu_{\lambda}^{(j)}\right)\right\}_{j \geq 0, \lambda \in K}=\left\{\left(\mu_{q}^{(j)}, \mu^{(j)}\right)\right\}_{j \geq 0, q \in K}$,

- $\left\{\beta_{\lambda}\right\}_{\lambda \in \Lambda}=\left\{\tau_{\mu}^{\prime}(q)\right\}_{q \in K}$,

- for every $j \geq 1$ and for $n \geq 1, V_{n}^{(j)}=C b^{M n^{3 / 4} \log (n)} b^{-\beta n \varepsilon_{n}^{2}}$,

- for every $j \geq 1, \psi_{j}(\widetilde{\eta})=\mathcal{S}_{j}=[\exp (\sqrt{\alpha \log (j)})]$.

With these parameters the conditions of Proposition 2 are fulfilled. As a consequence, with probability one, for $j$ large enough, for all $q \in K$, $G S\left(\mu_{q}^{(j)}, \mu^{(j)}, \tau_{\mu}^{\prime}(q), N, \widetilde{\varepsilon}\right) \leq[\exp (\sqrt{\alpha \log (j)})]$.

Let us then consider the families $\left\{\left(\mu_{q}^{(j)}, \mu_{q}^{(j)}\right)\right\}_{j \geq 0, q \in K}$ and $\left\{\tau_{\mu}^{\prime}(q) q-\right.$ $\left.\tau_{\mu}(q)\right\}_{q \in K}$ instead of the family $\left\{\left(\mu_{q}^{(j)}, \mu^{(j)}\right)\right\}_{j \geq 0, q \in K}$ and $\left\{\tau_{\mu}^{\prime}(q)\right\}_{q \in K}$ respectively, and keep the same definitions for the other variables involved in Proposition 2 Then the same control as above holds for the growth speed $G S\left(\mu_{q}^{(j)}, \mu_{q}^{(j)}, q \tau_{\mu}^{\prime}(q)-\tau_{\mu}(q), N, \widetilde{\varepsilon}\right)$. Notice that here the vector $V^{(j)}$ is deterministic.

5.3. Proof of Theorem 3. Fix $\alpha>1$ and $K$ a compact subinterval of $J$ and $N=0$. A standard Markov inequality (as in Proposition 1) shows that for $j \geq 0, n \geq 1$ and $q \in K$, one has $\mathcal{N}_{n}\left(\mu^{(j)}, \tau_{\mu}^{\prime}(q), \varepsilon_{n}\right) \leq$ $b^{-n \tau_{n}^{(j)}(q)} b^{n q\left(\tau_{\mu}^{\prime}(q)+\operatorname{sgn}(q) \varepsilon_{n}\right)}$, where $\operatorname{sgn}(q)$ stands for the sign of $q$. Then, by Proposition 4 with probability one, one has for $j$ large enough, for $n \geq \exp (\sqrt{\alpha \log (j)})$ and for $q \in K$

$$
\mathcal{N}_{n}\left(\mu^{(j)}, \tau_{\mu}^{\prime}(q), \varepsilon_{n}\right) \leq b^{-n \tau_{n}^{(j)}(q)} b^{n q\left(\tau_{\mu}^{\prime}(q)+\operatorname{sgn}(q) \varepsilon_{n}\right)} \leq b^{n\left(\tau_{\mu}^{\prime}(q) q-\tau_{\mu}(q)+\varepsilon_{n}^{\prime}\right)},
$$

where $\varepsilon_{n}^{\prime}=\sup _{q \in K} M_{K} n^{-1 / 4} \log (n)+|q| \varepsilon_{n}$. One remarks that $\varepsilon_{n}^{\prime} \leq \varepsilon_{n}(1+$ $\left.\sup _{q \in K}|q|\right)$ for $n$ large enough. On the other hand, let

$$
E=\left(E_{\tau_{\mu}^{\prime}(q),[\exp \sqrt{\alpha \log (j)}]}^{\mu^{(j)}}(0, \widetilde{\varepsilon})\right) \bigcap\left(E_{\tau_{\mu}^{\prime}(q) q-\tau_{\mu}(q),[\exp \sqrt{\alpha \log (j)}]}^{\mu_{(j)}^{(j)}}(0, \widetilde{\varepsilon})\right) .
$$


Using Theorem 2] with probability one, for $j$ large enough, for all $q \in$ $N, \mu_{q}^{(j)}(E) \geq\left\|\mu_{q}^{(j)}\right\| / 2=1 / 2$. But, looking back at the definition of $E$, one remarks that $\mu_{q}^{(j)}(E) \leq \mathcal{N}_{n}\left(\mu^{(j)}, \tau_{\mu}^{\prime}(q), \varepsilon_{n}\right) b^{-n\left(\tau_{\mu}^{\prime}(q) q-\tau_{\mu}(q)-\varepsilon_{n}\right)}$ for $n \geq \exp (\sqrt{\alpha \log (j)})$, that is $b^{n\left(\tau_{\mu}^{\prime}(q) q-\tau_{\mu}(q)-\varepsilon_{n}\right)} / 2 \leq \mathcal{N}_{n}\left(\mu^{(j)}, \tau_{\mu}^{\prime}(q), \varepsilon_{n}\right)$.

\section{REFERENCES}

[1] V.I. Arnol'd, Small denominators and problems of stability of motion in classical and celestial mechanics, Ups. Mat. Nauk. 18 (1963), 91-192, English transl. in Russian Math. Surveys 18 (1963), 85-191.

[2] J. Barral, Continuity of the multifractal spectrum of a random statistically selfsimilar measure, J. Theoretic probab. 13 :1027-1060 (2000).

[3] J. Barral, F. Ben Nasr, J. Peyrière, Comparing multifractal formalisms: The neighboring boxes conditions, Asian J. Math. 7:149-165 (2003).

[4] J. Barral, M.O. Coppens, B.B. and Mandelbrot, Multiperiodic Multifractal Martingale Measures, J. Math. Pures Appl. 82:1555-1589 (2003).

[5] J. Barral, S. Seuret, Combining multifractal additive and multiplicative chaos, accepted for publication in Commun. Math. Phys. (2004).

[6] J. Barral, S. Seuret, Sums of Dirac masses and conditioned ubiquity, C. R. Acad. Sci. Paris, Ser. I 339: 787-792 (2004).

[7] J. Barral, S. Seuret, Growth speed in singularity sets of Mandelbrot cascades, submitted.

[8] J. Barral, S. Seuret, The multifractal nature of heterogeneous sums of Dirac masses, submitted.

[9] J. Barral, S. Seuret, Lévy processes in multifractal time and fixed points of the smoothing transformation, submitted.

[10] J. Barral, S. Seuret, Heterogenuous ubiquitous systems and Hausdorff dimension in $\mathbb{R}^{d}$, submitted.

[11] G. Brown, G. Michon, and J. Peyrière, On the multifractal analysis of measures, J. Stat. Phys. 66(3-4):775-790 (1992).

[12] M.M. Dodson, Exceptional sets in dynamical systems and Diophantine approximation. Rigidity in Dynamics and Geometry (Cambridge, 2000), 77-98, Springer (2002).

[13] A.H. Fan, Multifractal analysis of infinite products, J. Stat. Phys. 86(5/6):13131336 (1997).

[14] D.-J. Feng, E. Olivier, Multifractal analysis of weak Gibbs measures and phase transition - application to some Bernoulli convolutions. Ergodic Theory Dynam. Systems 23:1751-1784 (2003).

[15] T.C. Halsey, M.H. Jensen, L.P. Kadanoff, I. Procaccia, B.I. Shraiman, Fractal measures and their singularities: The characterization of strange sets, Phys. Rev. A, 33(2):1141-1151 (1986).

[16] Y. Heurteaux, Estimations de la dimension inférieure et de la dimension supérieure des mesures, Ann. Inst. H. Poincaré Probab. Statist. 34:309-338 (1998).

[17] S. Jaffard, The multifractal nature of Lévy processes, Probab. Theory Rel. Fields 114: 207-227 (1999).

[18] J.-P. Kahane, J. Peyrière, Sur certaines martingales de Benoît Mandelbrot, Adv. Math. 22:131-145 (1976)

[19] K. Khanin, Y. Kifer, Thermodynamic formalism for random transformations and statistical mechanics, in Amer. Math. Soc. Transl. (2) 171:107-140 (1996). 
[20] Y. Kifer, Fractals via random iterated function systems and random geometric constructions. Fractal geometry and stochastics (Finsterbergen, 1994), 145-164, Progr. Probab., 37, Birkhuser, Basel, 1995.

[21] Y. Kifer, Limit theorems for random transformations and processes in random environments, Trans. Amer. Math. Soc., 350: 1481-1518 (1998).

[22] M. Ledoux, M., Talagrand, Probability in Banach Spaces, Springer-Verlag, NewYork.

[23] J. Lévy Véhel, R.H. Riedi, TCP traffic is multifractal: a numerical study, INRIA research report, RR-3129 (1997).

[24] B.B. Mandelbrot, Intermittent turbulence in self-similar cascades: divergence of hight moments and dimension of the carrier, J. Fluid. Mech. 62:331-358 (1974).

[25] B.B. Mandelbrot, Fractals and Scaling in Finance: Discontinuity, Concentration, Risk, Springer (1997).

[26] G. Michon, Mesures de Gibbs sur les Cantor Réguliers, Ann. Inst. Henri Poincaré: Phys. Théor. 58:267-285 (1993).

[27] J.-F. Muzy, E. Bacry, Multifractal stationary random measures and multifractal random walks with log-infinitely divisible scaling laws, Phys. Rev. E 66: (2002).

[28] L. Olsen, A multifractal formalism, Adv. Math. 116:92-195 (1995).

[29] Pesin, Y., Weiss, H., The multifractal analysis of Gibbs measures: motivation, mathematical foundation, and examples, Chaos 7(1), 89-106 (1997)

[30] W. Philipp, W. Stout, Almost sure invariance principles for partial sums of weakly dependent random variables, Mem. Amer. Math. Soc. 161 (1975).

[31] D.A. Rand, The singularity spectrum $f(\alpha)$ for cookie-cutters, Ergod. Th. Dynam. Sys. 9:527-541 (1989).

[32] R. Riedi, An improved multifractal formalism and self-similar measures, J. Math. Anal. Appl. 189:462-490 (1995).

[33] R. Riedi, Multifractal processes, Long Range Dependence: Theory and Applications, eds. Doukhan, Oppenheim and Taqqu, (Birkhäuser 2002), pp 625-715.

[34] G. Zohar, Large deviation formalism for multifractals, Stoch. Proc. Appl. 79:229242 (1999).

inRia Rocquencourt, Équipe "Complex", Domaine de Voluceau Rocquencourt, 78153 Le Chesnay cedex, France 